\title{
Predicting Material Defects in Reactive Polymeric Flows
}

\author{
Francisco Chinesta
}

Laboratoire de Mécanique des Systèmes et des Procédés, UMR CNRS, ENSAMESEM, Paris, France 


\section{Introduction}

Reactive polymeric systems are widely used in foam blowing or in resin transfer molding (RTM) processes. Numerical modelling of reactive processes is very difficult because the flow kinematics is coupled with the chemical reacting kinetics, which depends mainly on the reaction time (time elapsed since the reactants' mixing). The kinematics - chemical kinetics coupling - is a key point in foam blowing, being less important for example in usual RTM processes, where the chemical formulation can be adjusted in order to finish the mold filling before a significant rise in the resin viscosity.

The polymerization reaction increases the material viscosity and the expansion induces the material growth, generating a porous structure. When the polymerization takes place much faster than the foam expansion, the material becomes too rigid before the conclusion of the expansion. In this case the cellular walls can be broken with direct consequences for the mechanical properties of the conformed pieces. On the other hand, when the expansion finishes before the complete polymerization, the low material consistency is not enough to preserve the final geometry, and the structure collapses when the expansion is finished.

The numerical modelling of forming processes involving the flow of chemicallyreacting polymeric foams requires taking into account the different problem scales. Thus, in industrial applications a macroscopic approach is suitable, whereas the macroscopic flow parameters depend on the cellular structure: porosity, size shape and orientation of the cells, cellular walls properties, etc. Moreover, the shape and orientation of the cells are induced by the flow during the foam expansion (induced anisotropy). We have analyzed the influence of process conditions on the cellular shape. Elongated cells were obtained by applying a traction or a shear in the material during its expansion (Chirivella et al., 2000).

This microscopic information can be introduced in a macroscopic model by means of a homogenization technique. Thus, at each point in the material domain we can associate a characteristic volume containing some cells, whose size, shape and orientation depend on the point considered. Then, from the matrix and gas viscosities, and the cellular structure, we can compute an equivalent viscosity tensor. However, some difficulties appear in the homogenization procedure: (1) sometimes surface tension cannot be neglected, which requires a very accurate description of the inclusion geometry as well as consideration of its effect on the homogenized model; (2) the behaviour of the matrix fluid becomes quickly non-Newtonian, and its non linear character makes difficult the application of usual homogenization techniques; (3) the reaction kinetics remain uncertain for usual industrial processes; (4) the numerical tracking of a cell (microscopic level) which grows and deforms into the material flow is today an open problem, which in spite of some promising works (Sussman et al., 1994, 1999; Sussman and Fatemi, 1999; Sussman and Puckett, 2000), important difficulties persist (Paredes, 2001); and (5) the physical mechanism of gas diffusion, the cells interaction and fusion, and the modelling of the 
final stage of the process, when the material is concentrated on the cell walls, are, among many others, important and unsolved difficulties found in the global modelling of foam growing.

In this way a macroscopic modelling where the reaction kinetics and the viscosity evolution are obtained experimentally is suitable from the point of view of its industrial application. Thus, such a numerical modelling could provide a visualization of mold filling (foam volume evolution and flow front position during the expansion reaction) and will be useful to optimize the mold and process design. From that simulation we can determine the number, size and optimal position of the evacuation orifices to avoid internal overpressures (reducing the cleaning necessities as well as the material losses). Moreover, an accurate flow front tracking allows us to predict the interactions between different flow fronts when the curing reaction is too advanced (welding lines are fragile) as well as to avoid filling defects due to the big air bubbles retained between the flow front and the mold walls.

\section{Simplified flow model}

In order to obtain a macroscopic simplified flow model, able to carry out fast simulations of reacting foam flows, we consider the usual balance equations:

- The equilibrium equation neglecting the inertia and mass terms gives

$$
\operatorname{Div} \underline{\underline{\sigma}}=\underline{0}
$$

where $\underline{\underline{\sigma}}$ is the stress tensor.

- The mass conservation

$$
\frac{\partial \rho}{\partial t}+\operatorname{Div}(\rho \underline{v})=0
$$

where $\underline{v}$ is the velocity field and $\rho$ the foam density. The previous equation can be rewritten as

$$
\frac{\partial \rho}{\partial t}+\rho \operatorname{Div} \underline{v}+\underline{v} \cdot \operatorname{Grad} \rho=0
$$

When the foam expansion is homogeneous $\rho=\rho(t)$ and Grad $\rho=\underline{0}$. In this case equation [3] becomes

$$
\operatorname{Div} \underline{v}=-\frac{\partial \rho}{\partial t} \frac{1}{\rho}=\beta(t)
$$


This function can be defined from the chemical reaction kinetics or from a simple experimental test. The first procedure, as previously discussed, remains very inaccurate because it is very difficult to know all the chemical reactions and their kinetics, in order to compute accurately the evolution of the density. Thus, we prefer, as proposed in Chinesta et al. (Chinesta et al., 2000), to proceed from a direct determination of the density evolution.

- The constitutive equation establishes the relation between the stress tensor $\underline{\underline{\sigma}}$ and the strain rate tensor $\underline{\underline{D}}$

$$
\underline{\underline{\sigma}}=\underline{\underline{\sigma}}(\underline{\underline{D}, t})
$$

where $2 \underline{D}=\operatorname{Grad} \underline{v}+(\operatorname{Grad} \underline{v})^{T}$. For compressible flows $\operatorname{Tr}(\underline{\underline{D}})=\operatorname{Div} \underline{v} \neq 0$.

Equation [5] indicates also that the foam rheology depends on the reaction time. The simplest constitutive relation consists of

$$
\underline{\underline{\sigma}}=-p \underline{\underline{I}}+\left(\lambda-\frac{2}{3} \eta\right) \operatorname{Tr}(\underline{\underline{D}}) \underline{\underline{I}}+2 \eta \underline{\underline{D}}
$$

where $\lambda$ denotes the volumetric viscosity, $p$ the pressure field, $I$ the unit tensor and $\eta$ the shear viscosity. If the volumetric viscosity contribution is neglected

$$
\underline{\underline{\sigma}}=-p \underline{\underline{I}}+2 \eta \underline{\underline{D^{\prime}}}
$$

where $\underline{\underline{D}}^{\prime}$ denotes the deviatoric strain rate tensor. The viscosity evolution of the matrix fluid can be measured using usual techniques (Richter and Macosko, 1980). The most difficult task is the determination of the foam viscosity evolution (when the foam is considered as a pseudo-homogeneous fluid). The rheological characterization is difficult on account of the impossibility to proceed with standard rheometers which are not adapted to multiphase flows where the cell size is of the same order as the diameter or the gap of capillary or cone-plate rheometers respectively. Thus, some authors propose the use a of viscosity law in the form of a mixing rule that includes as control variables the concentration in reactants, the shear rate, the temperature and the gas volume fraction (Lefebvre and Keunings, 1993). Other authors prefer to describe the foam rheology by means of a simple power law model (Deshpande and Barigou, 2001) which must be identified using a nonstandard device. The flow consistency and the behaviour indices of the power law equation could depend on time to describe the material polymerization.

- We can notice that thermal effects are not taken into account in the previous model in order to minimize the parameters which must be identified experimentally, although it is well known that the kinetics depends significantly on the temperature field (usually the determination of the reaction kinetics is made from the temperature evolution measured during the material curing). 


\section{Numerical modelling}

When the foam expansion is heterogeneous, the density depends on the spatial coordinates, and the mass conservation equation is expressed by equation [3]. In this case the variational formulation of equations [1], [3] and [6] results in

$$
\int_{\Omega(t)}\left(\left(-p+\left(\lambda-\frac{2}{3} \eta\right) \operatorname{Tr}(\underline{\underline{D}})\right) \operatorname{Tr}\left(\underline{\underline{D}}^{*}\right)+2 \eta \underline{\underline{D}}: \underline{\underline{D}}^{*}\right) d \Omega=0
$$

and

$$
\int_{\Omega(t)}-p^{*}\left(\operatorname{Div} \underline{v}-\beta+\frac{G r a d \rho}{\rho} \cdot \underline{v}\right) d \Omega=0
$$

The numerical discretisation of the flow model for an homogeneous foam expansion was discussed in a former work (Chinesta et al., 2000) From the kinematics point of view the discretisation of the flow equations governing the heterogeneous foam expansion, equations [8] and [9], do not introduce particular difficulties, if we can assign to each point in the foam domain and for each time the corresponding values of $\beta, \lambda, \eta$ and $\rho$. Usually, the volumetric viscous term can be neglected, $\beta$ derives directly from the density $\rho$, whose time evolution is obtained following the procedure described in the previous section. Finally, in some simple rheological models, the foam shear viscosity depends on the equivalent strain rate as well as on the time, due to the polymerization and blowing kinetics.

In this form, for each point in the fluid domain at any time, we need to know, for the fluid located at that point, the time elapsed from the beginning of the chemical reactions (reaction time). Knowing that time we can assign the density and viscosity values.

In the case of homogeneous expansions we assume that the reactants are dumped in the mould simultaneously, and in consequence the reaction time is the same for any point, which corresponds to the usual Eulerian time when we consider that the reaction starts at $t=0$.

However, the manufacture of big pieces requires a great amount of reactants that must be distributed in large areas. In this case, we can not neglect the time elapsed in the reactants' dumping, so that, sometimes, the blowing starts before the complete reactant dumping, giving rise to a heterogeneous foam expansion. In that case, we need to label each point of the reactant fluid volume with its dumping time $T_{d}$, and transport this field during the foam expansion. Thus, we must solve the following linear advection equation 


$$
\left\{\begin{array}{l}
\frac{d T}{d t}=\frac{\partial T}{\partial t}+\underline{v} G r a d T=0 \\
T(\underline{x}, t=0)=T_{d}(\underline{x})
\end{array}\right.
$$

For example, if we suppose that reactants are dumped over a plate of one meter, from the left $(x=0)$ to the right $(x=1)$, in one second, the time label $T_{d}$ could be for example $T_{d}=x-1$. If we take the origin of time $(t=0)$ when the total amount of reactants is dumped, the reaction time $t_{r}$ will be given by $t_{r}=t-T$.

When an Eulerian technique, using a fixed mesh, is adopted to solve equation [10] some difficulties appear, related to the field updating in elements whose filling stage is just starting. To illustrate this fact we consider an empty finite element at time $t$, which starts its filling process. In this case, as we are using a fixed mesh strategy, the field $T$ updating in an element $\Omega^{e}$, i.e. the computation of $T^{e}$ at time $t+\Delta t$, makes use of its value at time $t$. Because this element starts its filling process at time $t$, the field $T(t)$ is not defined. If the simulation is carried out without paying attention to the different field initializations, great deviations appear, mainly in the neighborhood of the flow front, whose incidence cannot be neglected. For example, in the welding of several flow fronts we require active chemical kinetics to assure the material cohesion. In order to predict this kind of material defect, we need carry out simulations taking into account the hyperbolic character of advection equation, keeping good accuracy in the flow front treatment.

The main original contribution of this work is the establishment of a new general strategy able to carry out the field transport accurately even through the flow front. This technique minimizes the numerical diffusion and implicitly transports all fields with the flow front movement. The proposed technique can be also applied to general models involving advection or advection-diffusion equations.

\section{Transport problems}

As discussed in the last section, a transport problem appears associated with the advection of the field $T$, which is governed by the following linear advection equation [10]. If we consider a first order discontinuous finite element discretisation then

$$
T^{e}(t+\Delta t)=T^{e}-T^{e} \frac{\Omega^{+}}{\left|\Omega^{e}\right|}+T^{e^{-}} \frac{\Omega^{-}}{\left|\Omega^{e}\right|}+T^{e} \beta \Delta t
$$


where $T^{e}(t+\Delta t)$ is the value of $T$ in the element $\Omega^{e}$ at time $t+\Delta t, \Omega^{-}$the fluid volume coming to the element $\Omega^{e}$ from the upstream element $\Omega^{e^{-}}$(where the value of $T$ is given by $T^{e^{-}}$), $\Omega^{+}$is the fluid volume leaving the element $\Omega^{e}$ and $\Omega^{e} \mid$ is the volume of $\Omega^{e}$.

A first problem appears suddenly if we consider the time $t$ for which an element $\Omega^{e}$ starts its filling process from its upstream element $\Omega^{e^{-}}$. To illustrate this limitation we consider the simplest situation of an incompressible fluid, i.e. $\beta=0$. In this case, with $\Omega^{+}=0$, we obtain

$$
T^{e}(t+\Delta t)=T^{e}+T^{e^{-}} \frac{\Omega^{-}}{\left|\Omega^{e}\right|}
$$

which establishes that solution at $t+\Delta t$ is dominated by the solution existing in the element at time $t$, at which this element is empty, and consequently the property $T$ is not defined. The question is: what is the value to assign to $T$ before the beginning of its filling process?

An accurate and consistent discrete form of the conservation equation can be obtained by considering a discontinuous finite element discretisation of the equation which results from the addition of equation [10] multiplied by the fluid fraction $I$, whose evolution is governed by the advection equation (Chinesta et al. 2000)

$$
\frac{d I}{d t}=\frac{\partial I}{\partial t}+\underline{v} G r a d I=0
$$

with equation [13] multiplied by the field $T$, as proposed in Chinesta et al. (Chinesta et al., 2002)

$$
\frac{d(I T)}{d t}=\frac{\partial(I T)}{\partial t}+\underline{v} \operatorname{Grad}(I T)=0
$$

which results in

$$
\begin{aligned}
& T^{e}(t+\Delta t)=\sum_{i} T^{e^{-}} \frac{\Omega_{i}^{-}}{I^{e}(t+\Delta t)\left|\Omega^{e}\right|}+ \\
& +T^{e}\left(\frac{I^{e}(t)}{I^{e}(t+\Delta t)}-\frac{\Omega^{+}}{I^{e}(t+\Delta t)\left|\Omega^{e}\right|}\right)+\frac{\beta T^{e} I^{e}(t) \Delta t}{I^{e}(t+\Delta t)}
\end{aligned}
$$


where $\Omega_{i}^{-}$is the volume of fluid coming to $\Omega^{e}$ from the upstream element $\Omega^{e_{i}^{-}}$; with

$$
\begin{aligned}
& I^{e}(t+\Delta t)=I^{e}+\sum_{i} I^{e_{i}^{-}} \frac{\Omega_{i}^{-}}{\left|\Omega^{e}\right|}-I^{e} \frac{\Omega^{+}}{\left|\Omega^{e}\right|}+\beta I^{e} \Delta t= \\
& =I^{e}+\sum_{i} \frac{\Omega_{i}^{-}}{\left|\Omega^{e}\right|}-\frac{\Omega^{+}}{\left|\Omega^{e}\right|}+\beta I^{e} \Delta t
\end{aligned}
$$

\section{Numerical example}

We consider a numerical example involving a welding line development from two reacting flows with different reaction times. Figure 1 shows the foam presence function and the velocity field at three steps in the mold filling. Figure 2 depicts the transport of the dumping time. The actualization of the field $T$ is carried out by using equations [15] and [16].
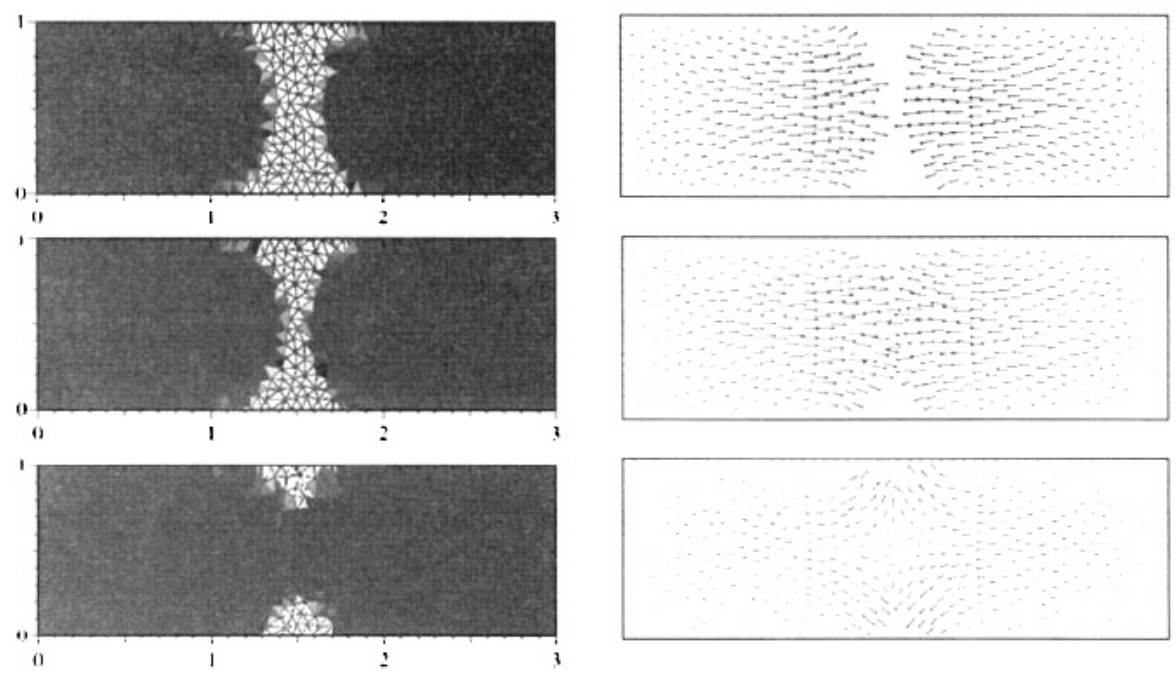

Figure 1. Foam presence and velocity field during the expansion and welding 

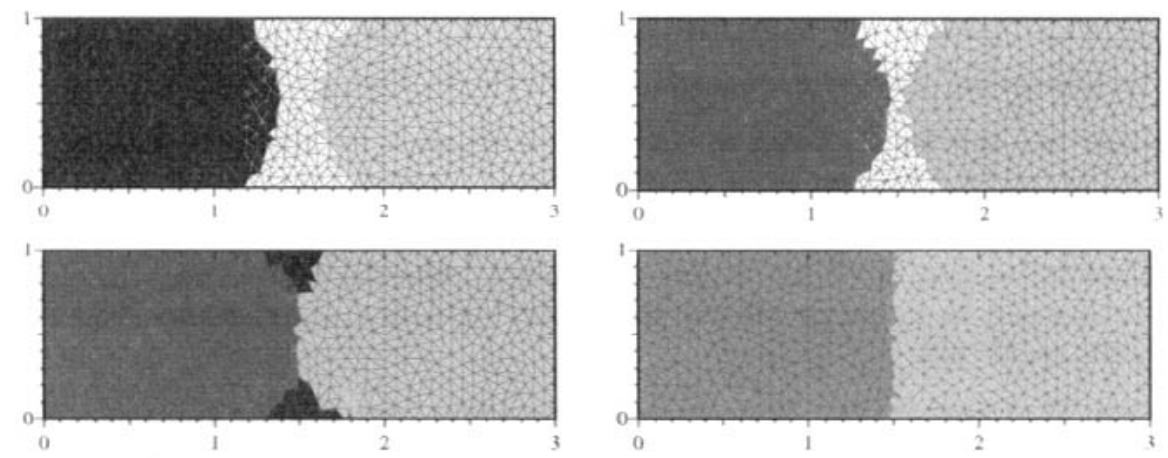

Figure 2. Transport of the dumping time and welding line development

\section{Conclusions}

From these results we can draw conclusions about the accuracy and low numerical diffusion of the numerical strategy proposed in the discretisation of equation [10]. The results are significantly improved, in relation to standard discontinuous finite element discretisations, mainly in the neighborhood of the flow front. In this way the proposed strategy can be used to compute accurately the reaction times in welding areas in 3D foam expansion process simulation, to predict defects in the material welding.

\section{Bibliography}

Chirivella P., Chinesta F., Godet M., Experimental analysis of the induced anisotropy in foam forming processes, Internal report LRTMM, CNAM Paris, 2000.

Chinesta F., Chaidron G., Godet M., Bermudez A., "Evaluation d'un modèle numérique simplifié de l'expansion de mousses", Les Cahiers de Rhéologie, Vol. 17, No. 1, 2000, pp. 403-412.

Chinesta F., Mabrouki T., Ramon A., "Some difficulties in the flow front treatment in fixed mesh simulations of composites forming processes", $5^{\text {th }}$ International Esaform Conference, Krakow, 2002.

Deshpande N.S., Barigou M., "Foam phenomena in sudden expansion and contractions", International Jour. of Multiphase Flow, Vol. 27, 2001, pp. 1463-1477.

Lefebvre L., Keunings R., "Mathematical simulation of the flow of chemically-reacting polymeric foams", Int. Conf. on Mathematical Modelling for Material Processing, Clarendon Press, 1993, Oxford, pp. 399-417.

Paredes J., Numerical simulation of the bubbles movement inside a flow (spanish), Internal rapport, Departamento de Matematica Aplicada, Universidad de Santiago de Compostela, Spain, 2001. 
Richter E.B., Macosko C.W., "Viscosity changes during the isothermal and adiabatic network polymerization", Polymer Eng. and Sci., Vol. 20, No. 14, 1980, pp. 921-924.

Sussman M., Smereka P., Osher S., "A level set approach for computing solutions to incompressible two-phase flows", Journal Comput. Physics, Vol. 114, 1994, pp. $146-$ 159.

Sussman M., Almgren A., Bell J., Colella P., Howell L., Welcome M., "An adaptative level set approach for incompressible two-phase flows", Journal Comput. Physics, Vol. 148, 1999 , pp. 81-124.

Sussman M., Fatemi E., "An efficient interface preserving level set redistancing algorithm and its applications to interfacial incompressible fluid flows", SIAM J. Sci. Comput. Vol. 20, No. 4, 1999, pp. 1165-1191.

Sussman M., Puckett E., "A coupled level set and volume-of-fluid method for computing 3D and axysymmetric incompressible two-phase flows", Journal Comput. Physics, Vol. 162, 2000 , pp. 301-337. 